\title{
Sustainable development indicators for integrated coastal management: definition area and spatial properties
}

\author{
J. Kaulins, R. Ernsteins \& I. Kudreņickis \\ Department of Environmental Management, University of Latvia, Latvia
}

\begin{abstract}
When designing a sustainability indicator system (SIS) within the integrated coastal management (ICM) development process, there are still to be encountered a number of difficulties in the constructing of the system both in the indicator selection process and later when analysing the system and assessing its degree of correspondence to the direct objective of application, reflecting the set of planning targets and accounting for the specifics of the particular coastal territory. During research and development work in the coastal municipalities in Latvia, particularly, in Saulkrasti municipality, there was developed municipal level SIS, being locally discussed and acknowledged by experts and municipality specialists. Saukrasti SIS was developed, based on the adaptation of the known theoretical approaches and previous national case studies research done. This practice based experience also gave an inspiration for a number of theoretical generalisations about coastal indicators definition area, their spatial properties and, especially, algorithmic scheme for designing sustainability indicators.
\end{abstract}

Keywords: indicators, algorithm, coastal zone, integrated management, sustainability capitals, spatial, governance

\section{Introduction to indicators definition}

In the literature on measuring sustainable development, a number of sustainability indicator definitions by different authors and institutions can be found being based mainly on two approaches: conceptual and functional [1].

As the name suggests, conceptual definitions are derived from the indicator concept itself and are important for understanding it. According to FAO definition [2], an indicator is defined as a variable, pointer, or index related to a 
criterion. Its fluctuations reveal the variations in those key elements of sustainability in the ecosystem, the fishery resource or the sector and social and economic well-being. The position and trend of an indicator in relation to reference points or values indicate the present state and dynamics of the system. A more general explanation is given as by Garcia and Staples [3]: indicators are pointers that can be used to reveal or monitor conditions and trends in the fisheries sector and the marine environment. Similar definitions can also be found by Slocombe [4], Fletcher et al, [5], OECD, [6], however one of the most comprehensive definitions is offered by Maureen Hart: "An indicator is something that helps you understand where you are, which way you are going and how far you are from where you want to be. A good indicator alerts you to a problem before it gets too bad and helps you recognize what needs to be done to fix the problem. ... They allow you to see where the problem areas are and help show the way to fix those problems." [7].

All of the above definitions, however, answer to the question of ,Why do we need indicators?" rather than explains what it actually is. Functional definitions explain what exactly the indicator does and how it differs from a simple parameter or measurement. This type of explanations is provided by Garcia et al [3], Smeets and Weterings [8], Hak et al., [19]). From the definitions analysed, the following one, approved by the United Nations Council on Sustainable Development in 2001, has been selected as the most comprehensive:

Indicators for sustainable development are in order to: 1. translate physical and social science knowledge into manageable units of information that can facilitate the decision-making process; 2. help to calibrate and measure progress towards sustainable development goals; 3. provide early warning to prevent damage; and 4. communicate ideas, thoughts and values (cited from Brown, Reyntjens [1]).

However, when constructing a specific indicator system, the practical use of this definition is cumbersome, as it fails to give a clear answer as to whether the selected value corresponds to indicator specifics or not. To receive such an answer, the indicator definition needs to answer to the following questions:

1) what values can serve as indicators, 2) what do these values characterise, 3) what is their role in the governance system, 4) what are the limitations of indicator functions, 5) what formal qualities distinguish an indicator from other values that can be measured, 6) what is the significance of the measurable parameter for a given management system. The definition cited earlier [1] answers to the first three of these questions and partially to number six as well.

Based on the above, we find the role of indicators in the hierarchical structure of management elements (Figure 1), which is built in line with the stages of the planning implementation cycle. Manageable units = measurable units; it means that an indicator must be expressed by numeric values. The same point also indicates to the principal role of indicators in the management system. Points 2 and 3 reflect a need for a string of successive measurements. Point 4, we consider, is more a conceptual one; to some extent, this also applies to Point 3. 


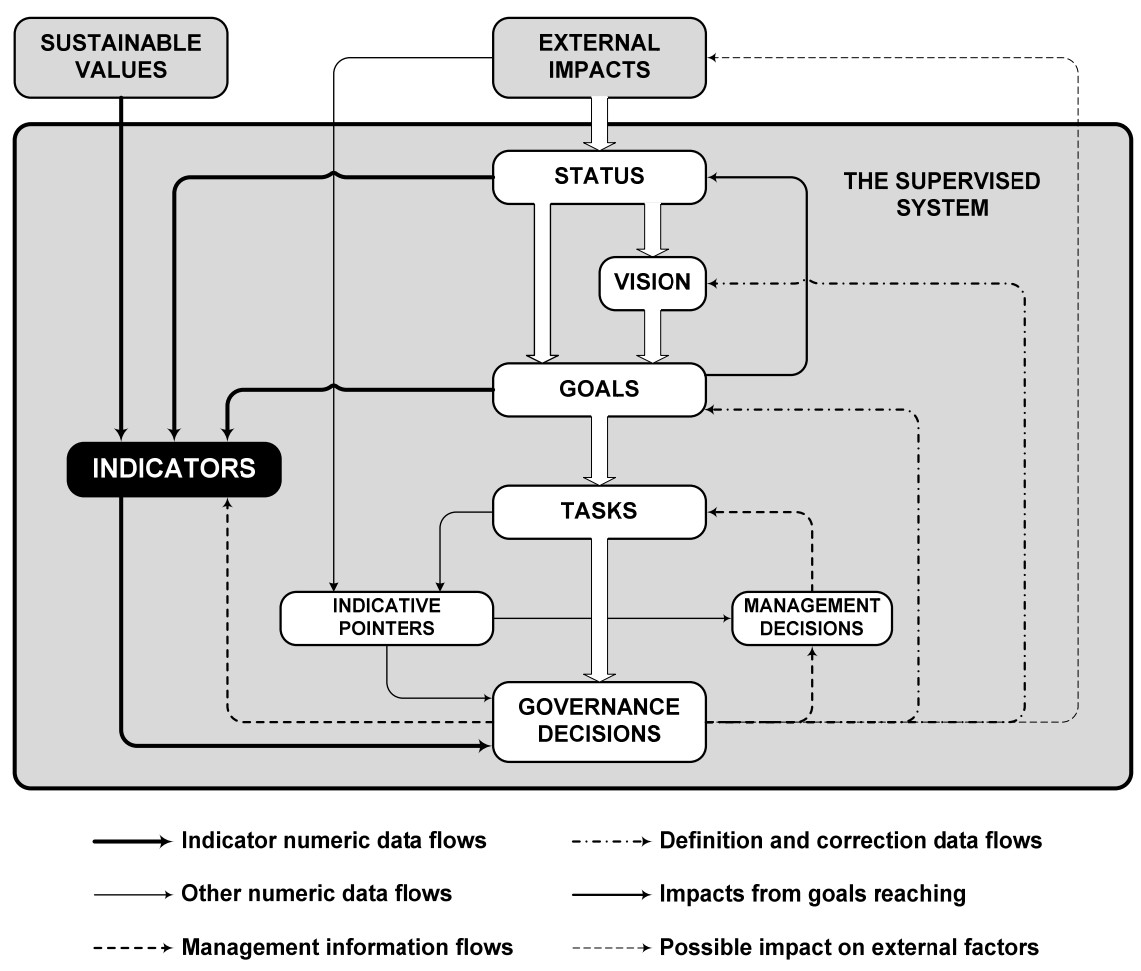

Figure 1: Indicators in planning.

Indicator applicability limits ensue from the above indirectly. A more specific description of it is provided by Sainsbury and Sumaila [10], defining that ,...an indicator that does not relate to an operational objective is not useful in this context". More specific conditions, however, are missing. A requirement for a formal feature cannot be found in the definitions mentioned; however, we find such indications in the latest literature: a datum or variable observed becomes an indicator only once its role in the evaluation of a phenomenon has been established [11]. In fact it means that relevant management decision is required. This also follows from the need for financial and human resource allocation for obtaining (and often accumulating) the data, doing the calculations and preparing the reports, which can take place only based on management decision.

\section{Definition area of sustainability indicators}

Indicators reflect the current situation as a point of reference on the one hand, and as advancement towards a strategically set planning goal on the other. If we consider a goal as a numerically defined value, it is easy to understand that it is located on a scale and can be corrected both within the current planning cycle and within new planning cycles to come. The tasks that are the means for reaching these goals cannot be placed on this scale, as in case of tasks, the 
attainment of the particular value characterises the end of the process, e.g., the use of financial resources allocated for a particular project. This also indicates to the fact that an indicator needs to function at the level of long-term goals. The need for assessing the importance of the measurable parameter ensues from practical considerations as well: the indicator method, when applied correctly, is expensive and complicated enough to use it for solving relatively generalised and long-term tasks only. To create an indicator system for the coastal sustainability long term monitoring, being based not only at the task level is very challenging and this certainly shall include then both indicators as we describe and also indicative pointers, giving additional insight into the status of particular management system, as well.

Based on the above considerations, the authors first agree on the role of an indicator within the hierarchical cycle of governance (Figure 1). The diagram shows that indicators refer to the hierarchical governance level of goals only, defining initial conditions, status with relation to goal implementation and dependence on our value system, if we speak of the values that we consider worth preserving, i.e., that are sustainability factors. Indicators may influence process governance through governance decision-making - by determining or adjusting this governance (and the goals themselves) in accordance with indicator readings. The notion of sustainable governance contains two subnotions: sustainability as the ability of the system to preserve the defined values, and governance as influence on this system. Accordingly, the definition of sustainable governance indicator should reflect both aspects. An attempt to do it in one definition could lead to complicated and miss-interpretable construction. Therefore it shall be divided into two parts: governance and sustainability.

A governance indicator illustrates a development factor or a set of factors and helps the public and decision-makers to get an impression of and control the situation with regard to the initial conditions from which the development goals defined in development planning documents ensue at the given governance level. In other words, a parameter or a group of parameters can be defined as a governance indicator if it/they reflect comparatively and unequivocally the numeric values of resulting indications concerning governance goals and changes in these values and allow for determination of the status and trends, and which can - directly or indirectly - be influenced significantly with decisions on the given governance level only.

A sustainability indicator reflects our perspectives on the values that are to be preserved in the name of our own and future generations: a parameter or a group of parameters can be defined as a sustainability indicator if it/they reflect comparatively and unequivocally the numeric values of sustainability impact factors and changes in these values and allow for determination of the status and trends, and which can - directly or indirectly - be influenced significantly with decisions on the given governance level only.

A parameter which at one governance level is an indicator is not necessarily an indicator at other levels; it follows from the presence of a governance level in the definition. A governance level here means the influence area within public administration: municipal, regional, national, etc. In case it is purposeful - for 
the sake of overall clarity - to show the values of such a parameter, this would then be an indicative pointer. It does not possess all functions of an indicator; that is, it does not reveal the efficiency of decisions taken. And vice-versa - a parameter which is only an indicative pointer at one level may become an indicator at other levels.

A phrase "...can be defined..." indicates the need for an administrative decision for a parameter to become an indicator. When applying the above definitions, an algorithm scheme for developing an indicator system may be constructed (see Fig. 2.). To be able to use the resultant product as a full-fledged indicator system, all indicators and indicative pointers need to have calculation methodologies developed and specific methods of result representation indicated.

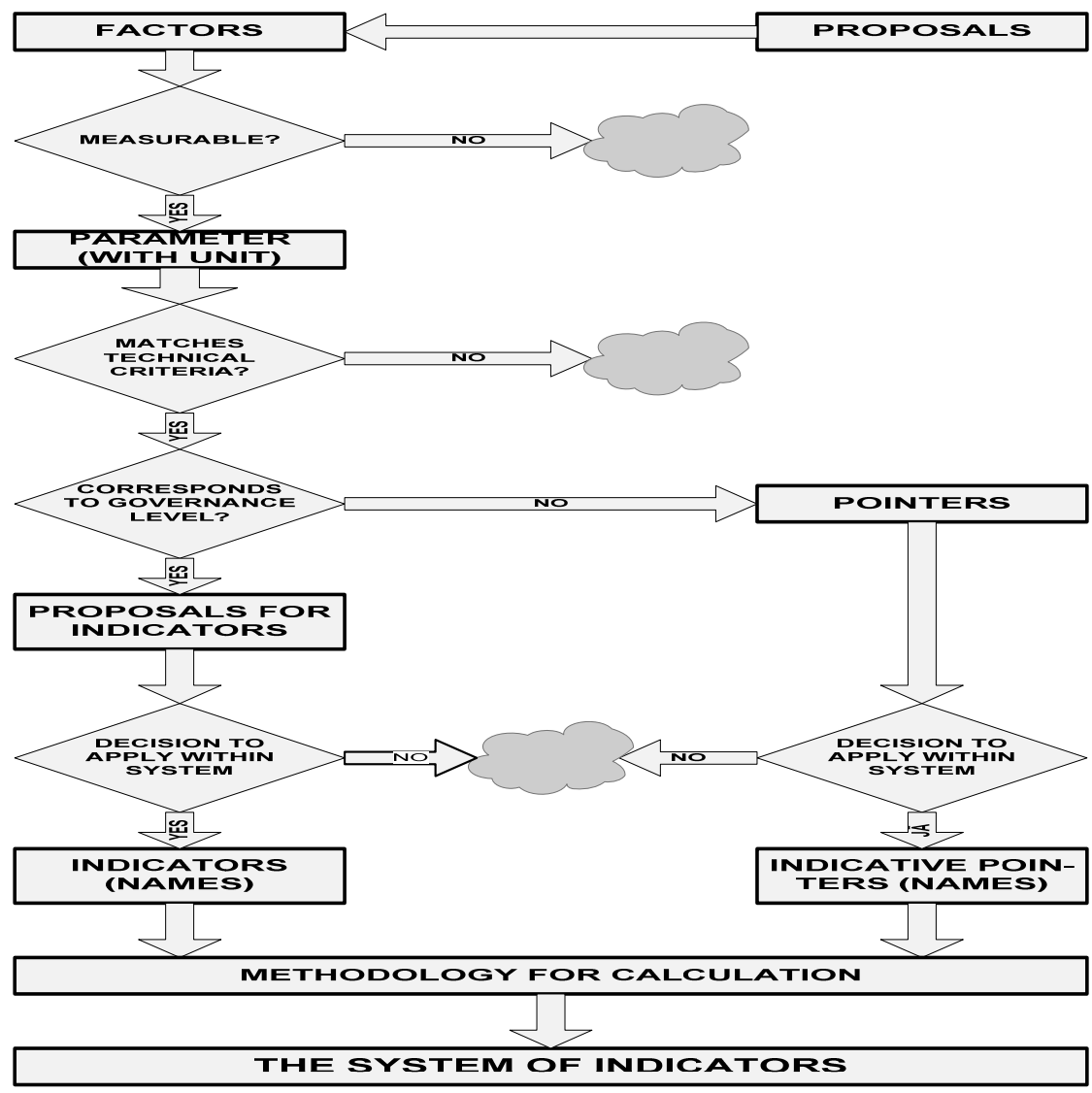

Figure 2: $\quad$ Algorithm for indicators.

Also, the following generic conditions are to be taken into account. Proposed factor is a parameter when it is measurable numerically. The factors proposed are in practice selected by various means: in system analysis, participatory within different target groups, following expert conclusions, through analysing existing 
systems as examples, etc. Also, parameter may not be used for constructing an indicator when its set of data does not meet the technical requirements regarding source data. This may not always be determined at this state; it sometimes manifests itself only when developing a methodology for calculations, or even worse - when indicator calculation is done for the first time. Parameter (or a group of parameters) becomes an indicator when it meets the technical requirements, is located within the indicator definition area and when a decision has been taken to apply it as an indicator. However, if a parameter meets the requirements but is not located within the indicator definition area, it may only be used as an indicative pointer.

From the above definitions, indicator utilisation limits, or the definition area in the respective governance system, also derive.

1. Given factor or set of factors F may serve as a territorial sustainable development indicator if it illustrates the implementation of sustainability goals defined in local governance planning documents and respective progress, and whose implementation and control falls within the competence of the given governance level. Labelling the governance level ' $i$ ' with $\mathrm{L}_{\mathrm{i}}$, the following law applies:

$$
\mathrm{F} \in \mathrm{L}_{\mathrm{i}}
$$

2. Given factor or set of factors $\mathrm{F}$ may not serve as a territorial sustainable development indicator if it only illustrates the values of an isolated planning task which is an intrinsic part of the set of goal-oriented measures and whose implementation applies unequivocally to a lower governance level competence:

$$
\mathrm{F} \in \mathrm{L}_{\mathrm{i}-\mathrm{n}} \text {, }
$$

where $\mathrm{n}-\mathrm{a}$ degree difference in governance levels; $\mathrm{n} \geq 1$.

3. Given factor or set of factors F may not serve as a territorial sustainable development indicator if the changes inflicted upon it by the given-level competence decisions $-\Delta \mathrm{F}_{\text {int }}-$ are small compared to the changes inflicted by a higher-level competence decisions $\Delta \mathrm{F}_{\text {ext }}$ :

$$
\left\{\begin{array}{c}
F \in L_{i+n} \\
\Delta F_{e x t}>\Delta F_{\text {int }} .
\end{array}\right.
$$

Based on the definitions and through assessment of indicator systems and their designing process, the concept of indicator integrativity was introduced, i.e., the range in which the particular indicator characterises a given governance system. The integrated management cycle planning applied in Saulkrasti municipality was based on municipal situation analysis in sustainability dimensions and on segmentation of priority integrative problem areas at dimension intersection points. The indicators were selected separately for characterising sustainability dimensions and also intergative problem areas.

By way of combining both resultant systems and assessing how the indicators refer to sustainability components, we can divide all indicators into 4 groups:

- Sub-sectoral indicators - describe an isolated, but governance levelspecific aspect of the respective sustainability dimension,

- sectorial indicators - principally describe one sustainability dimension,

- integrative indicators - describe integrative problem areas and other processes which concerns at least two sustainability dimensions, 
- integral indicators - describe the key, more general pointers of the governed system that characterise a given governance system in its entirety and/or compared to other similar systems.

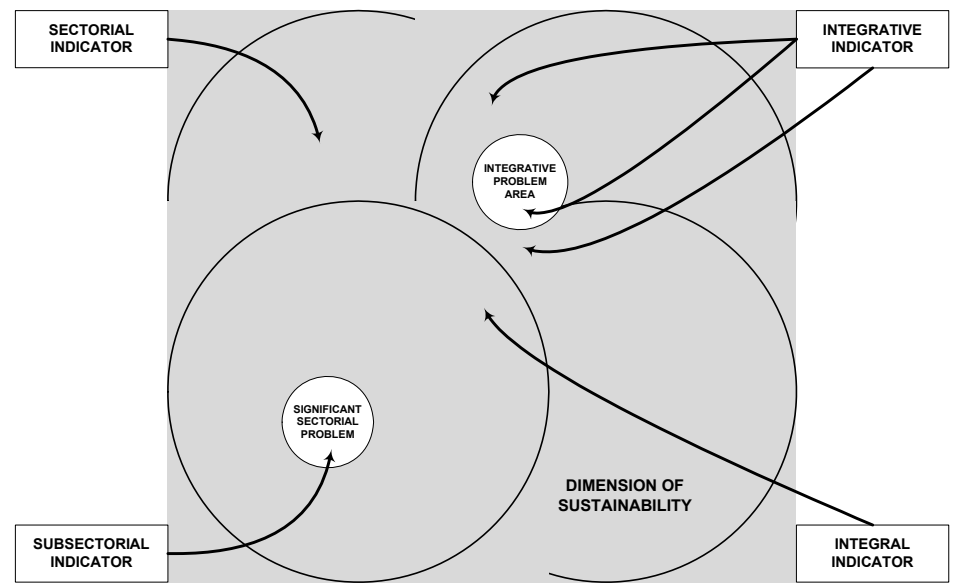

Figure 3: $\quad$ Indicator integrativity

The above division is to a certain degree similar to the one found in [12], but this source groups indicators according to target audience level pyramid principle and does not reflect the presence of the fourth dimension of sustainability - governance and communication environment. It can be understandable that the location of an indicator on the scale of integrativity levels generally correlates to the location in the target audience level pyramid, but this is not quite the same. Such integral indicators are fully possible which are significant or understandable only to expert audience (but required!), and an isolated sub-sectoral indicator may also characterise a very severe problem important at all levels - from the general public to experts and governance decision-makers. If both approaches are applied, it is convenient to analyse and assess the balance of the indicator system as per sustainability dimensions and to differentiate the contents of the material when drafting indicator reports and public reviews.

\section{Spatial properties of coastal sustainability indicators}

An indicator system for measuring coastal sustainability differs from the general case by its spatial specifics: the coastal zone is formed by a coastal line with the related set of other geospatial elements by Clark [13]. The indicator system though which coastal sustainability is assessed should therefore be able to at least differ the coastal zone from the inland and provide a comparison, to establish the origin of impact factors on the coastal status and development trends, and to create understanding of the distribution of coastal impacts within the governance territory. 
Ideally, the term 'coastal zone' should apply to a territory where the specific coastal impacts can be detected, and vice versa - a territory which impacts the developments on the coast and its proximity, as these impacts:

1) may in advance be unknown precisely enough,

2) may change over time,

3) differ for different factors,

4) The specifics of spatial distribution of the data used may prevent their correct differentiation.

In practice, the term 'coastal zone' is therefore applied to a relative territory which (see Fig.4) - within a single system - may in addition be applied in a number of ways depending on the data character.

Based specifically on the character of data spatial distribution, the EU project DEDUCE, which aimed at developing a European coastal sustainability model based on indicator system measurements, distinguished four key types of coastal zone definition (Marti X. et al, [14]):

- administratively territorial, which mainly represents the social and demographic information, as it is accumulated mainly concerning these aspects, with the local government as the smallest unit,

- co-ordinate character, which reflects locations of measurements in specific geographical co-ordinates (e.g., quality of surface waters) or in an object which is small enough for the range of measurements and can relatively be taken as a co-ordinate point (port operation) or is related to the geometrical coastline (coastline dynamics, artificial coastal constructions),

- a fixed-width coastal zone formed by the coastline's geometrical buffers; in the specific case differentiating between the nearby coastal zone $(1 \mathrm{~km})$ and the distant coastal zone $(10 \mathrm{~km})$; in this manner, geospatial information is assessed: land use, protected territories, etc.,

- combined, which contains the features of administrative territorial and coordinate points (e.g., number and location of objects in a specific territory); in this manner, thematic sectorial information is most often reflected such as the location of eco-certified tourist residences, etc.

There should be added, that comparison zone within local governments was defined as the territories of the districts of which at least $50 \%$ lie within the coastal $50 \mathrm{~km}$ buffer zone, and local governments in other districts of which at least $50 \%$ lie within the coastal $50 \mathrm{~km}$ buffer zone.

In our opinion, an additional fifth type is worth distinguishing on the local planning level, which by its data processing methods is similar to the geometrical but is related to spatial planning elements rather than buffers: protected zones and other types of territories with limitations of economic activities or other special regulations concerning the use of the particular territory.

The nature of data determines not only coastal definition types but also the spatial relations to the coast by indicators themselves. Here the following cases can be distinguished:

- special coastal indicators directly characterise some values characteristic of the coast only, e.g., catch of fish, bathing water quality, artificial coastal constructions, etc. 


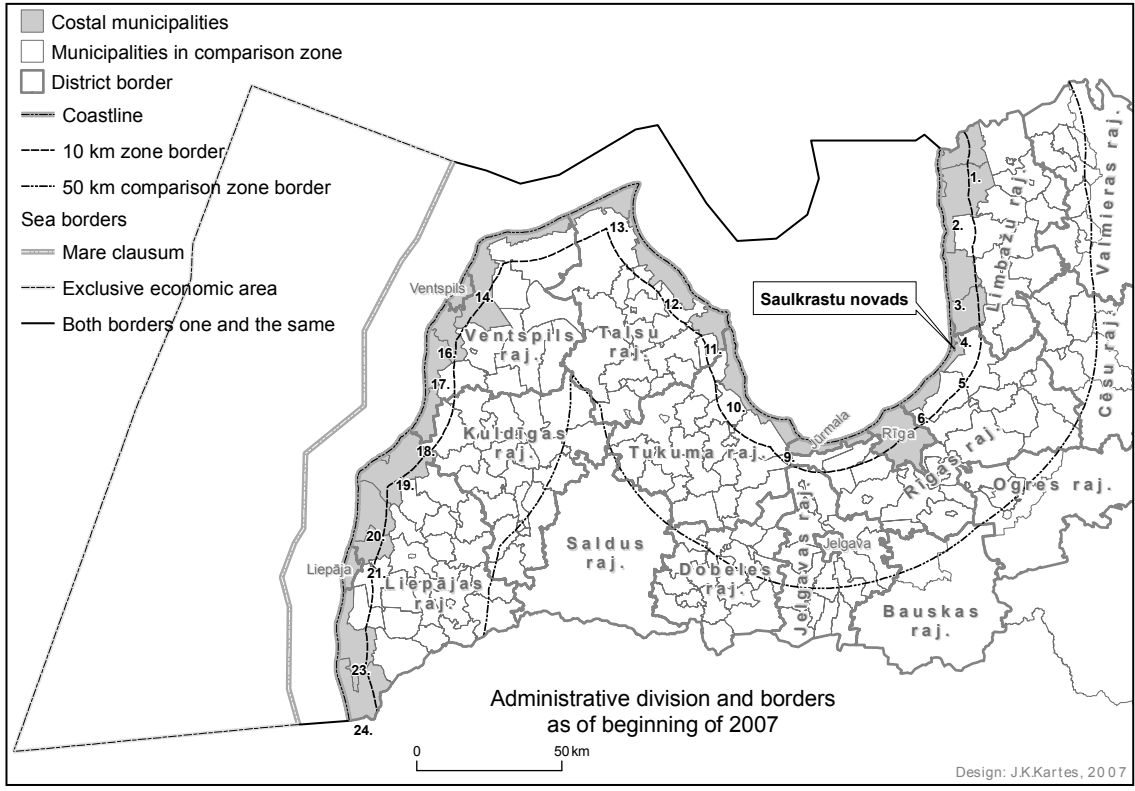

Figure 4: $\quad$ Coastal zone in Latvia.

- coastal discernible indicators which characterise elements not directly coastspecific but where a correct spatial assessment of the coast-related impacts is possible

- coastal relatively discernible coastal indicators, where the spatial distribution of data is "unclear", which prevents the correct determination of these impacts but our common knowledge about the territory allow for at least a qualitative assessment

- indicators non-applicable directly to the coast, which characterise a factor in the overall territory as a single inseparable object (e.g., number of residents, municipal budget values). However, here the coastal impacts can be assessed by mutually comparing such territories.

A strict line cannot be drawn between the discernible and relatively discernible parameters. It may in each case depend on data gathering and accumulation type. Also, when selecting the particular parameter as an indicator, we may plan changes in the data structure so as to improve coastal assessment opportunities in the periods to come.

This border also depends on the size of the local government. In national or international level indicator systems, the precision "to the municipality" is sufficient and even desirable, as in most cases easily available, safe and reliable statistical information is at hand $[13,14]$. In local planning, to be able to compare different parts of a territory and obtain information on coast-related impacts, more detailed elements need to be distinguished: isolated places of residence, land property (cadastre units), etc. In principle, this applies also to characterisation of spatial distribution of data origin (and availability). 
The developed classification helps us understand how large and in which areas and governance levels can the coast-specific impacts and processes be, and the role of governance decisions in these, as well as allowing for a more profound assessment of the importance of the impacts established as a result of measurements. There should be added, that according to Latvia legislature $5 \mathrm{~km}$ zone formal border is the geometrical border of a limited economic activity zone, which may be altered based on local geographic circumstances. The coastal dune protection zone is determined according to the Protected Zone Law: in villages $150 \mathrm{~m}$, outside $-300 \mathrm{~m}$ in width. The coastal geometrical buffer is altered by adding specially protected biotopes which adhere to the formal protected zone.

The above approach helps select the values to be measured which - in the current data availability - reflects most fully all the coastal impacts in their different aspects, and to receive significant additional information for the interpretation of results. In addition, data sources may also be evaluated and where possible - the degree of detailed elaboration may be improved so as to increase the indicator's spatial resolution.

The outlined approach may be fruitful not only for the coastal area but in all areas where a factor with a spatial impact is present in principle: proximity of a large city, a state border, geographical obstacles, etc.

\section{Saulkrasti indicator system case study}

Saulkrasti county, which is a small, relatively urbanised (for Latvian conditions) Baltic Sea coastal territory (Fig. 3), had in 2009-2010 coastal integrated development guidelines elaborated for it within the University of Latvia COBWEB project (COBWEB [15]), as the central component of municipal integrated governance. Special attention was devoted to measuring sustainability, and for this purpose, a system of indicators was developed. The system was elaborated based on the analysis of four dimensions of sustainability (natural, social and economic environment, governance and communication) and integrative problem areas as defined on their points of intersection.

Initially, over 100 indicators were proposed. However, after a selection according to the algorithm as presented in Fig. 2, the resultant system contains 55 indicators which thematically form 24 groups and reflect the status of all four dimensions of sustainability and provides overall characterisation of sectors, integrative problem areas and municipality as a whole (number in brackets show number of indicators in given thematic group and percent of total number of indicators by sustainability dimensions):

I. Nature environment (total 15, 27\%): Green frame status (3), Potential loads on the environment from public utilities (5), Air quality and climate change factors (3), Surface water quality (1), Land use development (1), Nature risks (2).

II. Economic environment (total 14, 26\%): Economically active people (1), Municipal budget (1),Traffic routes (2), Skulte port development (4), Tourism characteristics (6).

III. Social environment (total 13, 25\%):Health care characteristics (2), Supporting for cultural environment (3), S3 Employment and entrepreneurship 
(2), Social care and social security (2), Education system characteristics (1), Social life quality (3).

IV. Governance and Communication (total 5, 9\%): Activities for environment maintenance (1), Information of society about environmental events (2), Activities in nongovernmental sector (2).

V. Integral indicators (total 8, 13\%): Number of inhabitants (1), Area development index (1), Area attractivity index (1), Opinions of society (5).

We can see that within the system, both the traditional dimensions of sustainability are equally represented, except, governance and communication as horizontal dimension introduced as having increasingly growing role in Latvian conditions particularly, since the measurement possibilities and process itself is more time and other resources consuming as often has been based on opinion pools. It is difficult to find pointers that meet indicator requirements which characterise governance and communication; these are therefore represented to a lesser degree. This drawback, however, is compensated by the integral pointers section, which, together with the other sections, reflects the efficiency of governance perhaps most clearly, without singling out the contribution of any particular dimension.

The prevalence of integrative indicators in the system (64\%) stems from the broad approach to planning, which is based exactly on such integrative perspective of seeing sustainability dimensions in their interactions. Directly integrated problem areas are decrypted by $39 \%$ of all indicators. Separate sectors (e.g., tourism) are singled out when the related issues is significant enough for the development and welfare of the entire territory. The integral indicators also include separate indicators selected to characterise particular dimensions, as these bear a considerably larger content load, but are overall designed to characterise resident attitudes and opinions. Their number is comparatively small; in case of a bigger proportion, there is a risk of obtaining too general information, which provides an insufficiently detailed picture for the purposes of practical action and decision-making. The usable data sources are mainly the information accumulated by state and municipal institutions, and opinion-polls of businessmen, NGOs and residents. In one case, the utilisation of the opportunities afforded by public monitoring is planned in one case.

Considering the role of the coast in Saulkrasti county, a conclusion may be made that the $34 \%$ of indicators bearing the load of clear coastal characteristics (special coastal and with clear resolution of coastal zone), may be insufficient for this purpose. In future, however, by means of improving data collection and accumulation in municipal institutions, this share could be enlarged towards coastal relatively discernable data.

The system has been discussed with municipal and planning experts and the wider public in seminars and focus group discussions and assessed as a practically implemental as Saulkrasti county strategic planning element, being generally balanced indicator system, which emphasizes the local specifics of Saulkrasti and includes the key general ones. 


\section{Conclusions}

The developed indicator definitions and indicator selection algorithm allows for the careful selection of parameters that correspond to indicator meaning and purpose. This helps eliminate errors which might occur by introducing to the system parameters inappropriate or insignificant to a given governance level. The assessment of indicator spatial characteristics and classification allows for building an indicator system, in which the impacts of a coastal or other spatial factor on the respective governance system are reflected as fully as possible. When developing an indicator system for sustainability assessment in local coastal municipalities like Saulkrasti county, application of suggested above selection of indicators and the observation of indicator spatial characteristics leads to the resultant system, being balanced in terms of both reflection of sustainability dimensions as well as common territorial characteristics and description of coastal impacts. This type of an indicator system provides the opportunity to not only monitor sustainability of a territory and associated changes but also to follow the governance processes and control implementation of the strategic objectives as set in development planning documents and ensure continuous information to decision-makers and the public at large.

\section{References}

[1] Brown, J., Reyntjens, D. (2005) INDECO: Indicators - An Overview Internal Paper for Discussion. 1st April 2005

[2] Indicators for sustainable development of marine capture fisheries. FAO Technical Guidelines for Responsible Fisheries no 8. FAO, Rome. 68p. 1999

[3] Garcia, S.M., Staples, D.J. and Chesson, J. The FAO guidelines for the development and use of indicators for sustainable development of marine capture fisheries and an Australian example of their application. Ocean and Coastal Management, No 43, pp537-556, 2000

[4] Slocombe D.S. (1999) Defining goals and criteria for ecosystem-based management. Environ. Mgmt. 22: 483 - 493

[5] Fletcher, W.J., Chesson, J., Fisher M., Sainsbury, K.J., Hundloe, T., Smith, A.D.M. and Whitworth B. National ESD Reporting Framework for Australian Fisheries, Canberra, Australia, 2002

[6] OECD Environmental Indicators - Development, Measurement and Use. Reference Paper, 2003

[7] Hart M. Sustainable Measures. http://www.sustainablemeasures.com

[8] Smeets E. and Weterings R. Environmental indicators: typology and overview. European Environment Agency Technical Report no 25. EEA, Copenhagen, 1999

[9] Hak T., et al. Indicator-based evaluation of interlinkages between different sustainable development objectives. Review report on the state-of-the-art in sustainable development indicators. EU SDI project, , p.10, 2007 
[10] Sainsbury K. and Sumaila U.R. Incorporating ecosystem objectives into management of sustainable marine fisheries, including 'best practice' reference points and use of marine protected areas. Reykjavik Conference on Responsible Fisheries in the Marine Ecosystem, FAO, Rome, 2001

[11] Tanguay G.A., Rajanson J., Lefebvre J.-F., Lanoie P. Measuring the sustainability of cities: An analysis of the use of local indicators. Ecological Indicators 10, 407-418, 2010

[12] Sustainable Development Indicators to monitor the implementation of the EU Sustainable Development Strategy. Brussels, 9.2.2005 SEC(2005) 161

[13] Clark R.J. Coastal zone management for the new century. Ocean \& Coastal Management, Vol. 37, No. 2, pp. 191-216, 1997.

[14] Marti X. et al., Indicators Guidelines To adopt an indicators-based approach to evaluate coastal sustainable development. DEDUCE, EU Interreg III project, 98p., 2007

[15] Ernšteins R., Kauliņš J., Kudreņickis I. Sustainable Coastal Development Indicator System Studies In Latvia. Sustainable Development and Planning IV , vol.2, Southampton, WIT Press, pp.653-664, 2009

[16] Project COBWEB: http://www.hyria.fi/cobweb/about_cobweb/, last reviewed 2011.01.15 